\title{
Los jóvenes y la prensa: hábitos de consumo y renovación de contenidos
}

\author{
Dra. María Arroyo Cabello \\ Universidad Católica San Antonio de Murcia (UCAM) \\ Msarroyo@pdi.ucam.edu
}

\section{Resumen}

El estudio de la relación entre los jóvenes y la prensa revela, por una parte, la tendencia a la baja en el consumo de periódicos de referencia y, por otra, la penetración creciente de la prensa gratuita en este sector de la población.

Este trabajo analiza las causas de esta realidad periodística y las consecuencias que tiene para la formación intelectual de nuestros jóvenes, y señala las propuestas de varios autores acerca de la renovación de contenidos y otras estrategias de comunicación.

\section{Abstract}

The study about the relations between joungs and press reveals, on one side, that the press is loosing audience, and on other side the growing success of the free journals.

This article analyzes the causes of this journalistic reality and the consecuences for the education of joungs, and shows the proposals of several authors about the renewal of contents and the strategies of communication.

Palabras claves: Consumo, prensa, jóvenes, contenidos, estrategias.

Key words: consumption, press, joungs, contents, strategies.

\section{INTRODUCCIÓN}

\footnotetext{
1 pesar de los Planes de Fomento a la Lectura de los últimos años, el consumo de prensa entre los jóvenes no ha crecido como se esperaba, si nos atenemos a los datos que vienen publicando los Informes Anuales de la Comunicación.
}

Según el Estudio General de Medios, el perfil de la audiencia de lectores de prensa diaria en España no ha variado sustancialmente en los años 90; sin embargo, hay que resaltar la progresiva pérdida de lectores jóvenes, tal y como aparece en el cuadro 1. 
Cuadro 1. Evolución de jóvenes lectores de prensa diaria (1990-1999)

\begin{tabular}{|c|c|c|c|c|c|c|c|c|c|c|}
\hline Edad & 1990 & 1991 & 1992 & 1993 & 1994 & 1995 & 1996 & 1997 & 1998 & 1999 \\
\hline $\begin{array}{c}14 \text { a } 24 \\
\text { años }\end{array}$ & 23,0 & 23,0 & 23,0 & 22,5 & 22,6 & 22,8 & 22,0 & 21,0 & 19,3 & 18,5 \\
\hline
\end{tabular}

Fuente: EGM/AIMC ${ }^{1}$ y elaboración propia

La situación sigue siendo preocupante, porque el consumo de prensa diaria entre los 14 y 24 años va descendiendo año tras año desde 1997, como podemos ver en el cuadro 2, que recoge el perfil de los lectores de la prensa diaria entre 1997 y 2004.

Cuadro 2. Evolución de jóvenes lectores de prensa diaria (1997-2004)

\begin{tabular}{|c|c|c|c|c|c|c|c|c|}
\hline Edad & 1997 & 1998 & 1999 & 2000 & 2001 & 2002 & 2003 & 2004 \\
\hline 14 a 19 & 9,2 & 8,5 & 7,8 & 7,3 & 6,5 & 6,0 & 6,0 & 6,3 \\
\hline 20 a 24 & 11,8 & 10,8 & 10,8 & 10,8 & 9,9 & 9,2 & 9,1 & 8,8 \\
\hline
\end{tabular}

Fuente: AIMC, EGM² y elaboración propia

Por otra parte, las cifras que AEDE daba en 2003 confirmaban el descenso desde 1997 de lectores entre 18 y 34 años, si bien detectaban una recuperación en la tasa de penetración de lectura de diarios entre mayores de catorce años, que pasó del 35,8 por ciento en 2002 al 37,3 por ciento en 2003.

Ahora bien, si bien es cierto que uno de cada cinco jóvenes de entre 14 a 24 años lee diariamente el periódico, también es verdad que se trata de diarios deportivos en su mayoría y que la prensa diaria gratuita está calando en este sector de la población ${ }^{3}$.

Por otra parte, los jóvenes todavía consideran de referencia informativa a la prensa tradicional. Reconocen su implicación cada vez mayor con Internet, pero expresan sus dudas en cuanto a la fiabilidad de las informaciones que reciben de

\footnotetext{
${ }^{1}$ Recogido por Francisco Iglesias en Marketing periodístico, Ariel Comunicación, Barcelona, 2001, p.52

2 Informe Anual de la Profesión Periodística 2005, Asociación de la Prensa de Madrid.

${ }^{3}$ Según el Estudio General de Medios (2004), los dos periódicos gratuitos Metro (con un 23\% de lectores de 14 a 24 años) y 20 Minutos (19,42\%) son los que tienen mayores proporciones de público joven.
} 
la red. Es decir, aún la prensa les ofrece mayor credibilidad, lo cual no quiere decir que los periódicos hayan logrado interesar a los lectores jóvenes. Ante esta evidencia cabe preguntarse: ¿por qué a los jóvenes no les atraen los periódicos?

Las causas son complejas: la principal es la falta de convivencia con el diario en los hogares, pero también la existencia de contenidos inadecuados para este tipo de público y el avance de las nuevas tecnologías como medio informativo. Es un hecho que pocos jóvenes leen noticias, pero cuando lo hacen las leen en Internet y en diarios gratuitos, que requieren menos esfuerzo que el periódico de información general. Según la WAN (Asociación Mundial de Periódicos), la proporción de menores de 35 años entre los lectores de gratuitos es el doble que en los demás títulos. Las condiciones de gratuidad, de adquisición sin pasar por el quiosco, y la lectura rápida que ofrecen estos productos, constituyen una excepción en la prensa diaria y un acicate para el consumo.

La atención que requiere la interpretación y valoración de un texto escrito siempre ha supuesto un hándicap para los periódicos, pero la juventud de hoy es un público más difícil para la prensa tradicional que la de otras épocas. Nacidos en la era audiovisual, la generación actual que ha crecido con el mando a distancia y el ratón en la mano tiene menos afición a leer. Los jóvenes están mayoritariamente desinteresados por el soporte papel y se resisten a comprar en el quiosco unos diarios centrados en el análisis político, mientras que los medios electrónicos les ofrecen una cultura más afín a sus necesidades. Para un público que maneja con más gusto el cursor y las posibilidades del periodismo interactivo -al que ahora puede acceder sin dificultad desde otro de nuestros recientes objetos tecnológicos de uso habitual: el teléfono móvil ${ }^{4}$-, la prensa convencional se ha quedado obsoleta.

Los jóvenes tienen dificultades para incorporarse a los consumidores de prensa diaria porque se les ofrecen productos más propios de la generación de la Guerra Fría que de la del atentado contra las Torres Gemelas de Nueva York y la masacre de Madrid $^{5}$. En este aspecto, la publicidad ha sabido atender a su tiempo la demanda de ese segmento de la población, mientras que la prensa no ha caído en la cuenta.

A partir de la revolución juvenil de 1968 a los jóvenes se les reconoce una identidad propia que les diferencia de los niños y de los adultos. Desde entonces tienen sus propias diversiones, ropa, música, viajes, amigos, horarios, lugares de reunión, etc. Mientras la publicidad vio en ellos potenciales consumidores, la prensa, por el contrario, no reparó en posibles lectores. Así, nos encontramos con que las revistas juveniles son recientes y los periódicos siguen sin ofertar unos contenidos que puedan interesar a la juventud. Como los periódicos están hechos

${ }^{4}$ EDO, C., Del papel a la pantalla. La prensa en Internet, Comunicación Social, Sevilla, 2002, p.97. También encontramos referencias en el libro de DÍAZ NOCI, J., y MESO, K., Periodismo en Internet. Modelos de prensa digital, Universidad del País Vasco, Bilbao, 1999.

${ }^{5}$ LÓPEZ, M., Nuevas competencias para la prensa del siglo XXI, Paidós, Barcelona, 2004 , p.46. 
por y para los adultos, la prensa es contemplada por ellos como algo propio de otro tiempo y de otra generación.

Internet, por el contrario, es su medio, a diferencia de los adultos, a los que les ha resultado difícil adaptarse. Los niños y adolescentes de nuestro país han incorporado los hábitos interactivos de forma natural a su vida cotidiana, como se constata en el Estudio sobre Audiencia Infantil/Juvenil de Medios en España $2004^{6}$.

En resumen, la actitud de los jóvenes ante la prensa está motivada, primero, por el escaso atractivo que ejercen para ellos los diarios de información general debido a unos contenidos demasiado "institucionales" y "oficialistas" y a un lenguaje alejado de la dinámica juvenil. En segundo lugar, tampoco es fácil crear hábitos de lectura entre quienes no los han heredado de sus mayores, circunstancia mucho más determinante que la del poder adquisitivo cuando se analizan las prácticas informativas de las nuevas generaciones. En este aspecto, algunas encuestas realizadas entre universitarios coinciden en señalar que el escaso consumo de prensa está ligado fundamentalmente a dos factores: el bajo o nulo arraigo familiar en el hábito de lectura y la falta de sintonía entre los valores dominantes de la juventud y aquellos que constituyen la agenda de los diarios.

\section{AYUDAS PARA EL CONSUMO DE PRENSA}

Con objeto de ganar lectores jóvenes la WAN ${ }^{7}$ (Asociación Mundial de Periódicos) puso en marcha dos proyectos, uno de Periódicos en la Escuela y otro de Jóvenes Lectores, con los que pretende fomentar la lectura de periódicos entre los menores. Convencida de la importancia de estos programas, en abril de 2003, la Asociación Mundial de Periódicos firmó con una empresa noruega, líder mundial en papel prensa, un acuerdo de cinco años para lanzar una serie de nuevos programas destinados a utilizar los periódicos y las revistas con fines educativos.

En Francia el Ministerio de Cultura aprobó un plan de ayuda a la prensa diaria, que cuenta con una partida de 3,5 millones de euros en los presupuestos de $2005^{8}$.

En nuestro país la AEDE y el Ministerio de Educación firmaron el Plan de Fomento de la Lectura 2001-2004, por el que el Ministerio se comprometía a potenciar la utilización de los diarios como instrumento de formación para los

\footnotetext{
${ }^{6}$ Presentado por la Asociación de Investigación en Medios de Comunicación (AIMC) que estudia las audiencias de medios.

${ }^{7}$ La WAN, con sede en París, es la organización mundial de la industria de la prensa que representa a 18.000 periódicos; incluye entre sus miembros a 72 asociaciones nacionales de periódicos, ejecutivos periodísticos individuales en 100 países, 13 agencias de prensa y nueve grupos de prensa regionales e internacionales. http://www.wan-press.org/print.php3?id_article=956

${ }^{8}$ Estas ayudas permiten a los colegios y a los institutos el uso gratuito de la prensa como soporte pedagógico; así como potenciar la venta de diarios en los institutos a una tarifa reducida; promocionar programas infantiles de televisión que instiguen a la lectura o favorecer en las escuelas la conexión a portales de diarios en Internet (Informe Anual de la Profesión Periodística 2004).
} 
alumnos y como medio para favorecer el hábito de lectura. En febrero de 2003 ambas partes aprobaron una serie de iniciativas encaminadas a la formación del profesorado a través de cursos en los que intervendrían los propios editores. El proyecto incluía desarrollar conjuntamente campañas de publicidad para incentivar la lectura a través de todos los medios de comunicación. Más adelante, se podría ampliar a las Comunidades Autónomas. Pero todavía no se conocen los resultados de este ambicioso plan.

Los editores españoles son conscientes de la necesidad de asegurar la reposición futura de su base de lectores y del gravísimo problema cultural que supone la carencia de los mismos. Quizás por ello, en diciembre de 2004, el presidente de AEDE José María Bergareche se refirió a la necesidad urgente de acometer planes de fomento de la lectura para jóvenes, ya que en el Informe Pisa ${ }^{9}$ de la OCDE sobre comprensión de lectura entre jóvenes de quince años España volvía a estar a la zaga, sólo por delante de Portugal, Italia y Grecia y a la par con Luxemburgo. A este respecto, recordaba Bergareche que entre las conclusiones del informe de la OCDE destacaba un dato preocupante: el 21 por ciento de los españoles de quince años no alcanzaba el nivel básico de lectura y comprensión de textos escritos, y además se habían perdido posiciones a nivel general con relación a 2000 .

En el último informe de la Unión Europea, el nivel académico de los alumnos españoles está muy por debajo del de Alemania, Gran Bretaña, Francia, Italia y, por supuesto, Finlandia. Y el índice de fracaso escolar de la Educación Secundaria alcanza el 40 por ciento, porcentaje sólo superado por Malta y Portugal.

En este sentido, la reducción del IVA de los periódicos desde el cuatro por ciento actual a la media comunitaria del dos por ciento e incluso cero, podría frenar esta situación. A propósito de ello, Bergareche manifestó en nombre de los editores que estarían dispuestos a financiar muchas de las medidas de apoyo a la lectura juvenil de periódicos con los ahorros que resultaran de una reducción inmediata del IVA $^{10}$.

Otra propuesta de la AEDE, que puede favorecer el consumo de prensa entre los jóvenes, es la petición al Gobierno para la creación de una asignatura que se denominaría "Comunicación" o "Comunicación social" en la enseñanza secundaria. Esta propuesta permitiría que los alumnos se habituaran a la lectura de prensa y lo hicieran de una forma crítica, además de ayudarles a interpretar los mensajes y a una mejor comprensión de los textos.

Con el fin de acercar la prensa a los jóvenes los editores españoles han impulsado algunas otras iniciativas dirigidas a promover la colaboración entre las empresas periodísticas y los centros educativos. El resultado ha sido que muchos periódicos se distribuyen gratuitamente en escuelas y universidades, e incluso los

\footnotetext{
${ }^{9}$ Programme for International Student Assessment

${ }^{10}$ Libro Blanco de la Prensa Diaria 2005.
} 
Los jóvenes y la prensa...

principales diarios intentan involucrar a los escolares en el proceso de elaboración de contenidos con cuadernillos específicos para ellos y con concursos que les animen a seguir con interés las informaciones del diario desde edades aún más tempranas.

Al mismo tiempo, la prensa de tirada nacional ha apostado por los universitarios y encartan en sus productos algunas páginas dedicadas a ellos, pese a la creciente competencia de semanarios de este tipo que aparecen en todas las universidades.

En este sentido, hace tiempo que los diarios españoles emprendieron una actualización en los contenidos de su agenda temática con la finalidad de captar al público juvenil. Desde 1995 El País edita un suplemento, que sale los viernes bajo el título de Tentaciones, con un diseño muy novedoso, ágil y con portadas muy atractivas en buen papel. La fórmula se basa en unos contenidos centrados en música, cine, viajes, teatro, danza y televisión. El suplemento incluye un apartado de servicios con mucha información sobre conciertos y espectáculos en general. También está abierto a colaboraciones ocasionales, como crítica de cine. Esto le ha supuesto al diario un incremento de ventas los viernes de un $17 \%$, pero no ha demostrado que los lectores sigan comprándolo el resto de la semana.

Otra apuesta dirigida a los colegios ha sido la del diario El Mundo. Desde 1999 el periódico de Pedro J. Ramírez publica, para los centros escolares que lo soliciten, el suplemento "Aula", un título pensado para los alumnos pero lleno de iniciativas y noticias atractivas para ellos. Su presentación es similar a la de los demás suplementos de El Mundo, es decir, muy visual. Sus contenidos están coordinados en el Newspapers in Education, un programa desarrollado con el asesoramiento de la Asociación Mundial de Periódicos.

Hasta ahora estas iniciativas no solucionan el problema, ya que los suplementos y páginas juveniles se han revelado como subproductos, que no necesariamente crean afición por el periódico entero ni invitan a leerlo a diario.

Al parecer los suplementos no han servido para incentivar el consumo de prensa, pero tampoco las promociones han conseguido nada. Con las ofertas los periódicos han dejado de ser informativos y culturales para convertirse en vendedores de los más variopintos objetos. La consecuencia es un mantenimiento artificial de la difusión a cambio de una erosión del valor del producto: la información. En cambio, es más interesante la propuesta del Ministerio de Cultura francés de regalar a los jóvenes, cuando cumplan 18 años, una suscripción gratuita de dos meses al diario que elijan.

Por lo que hace a nuestro país, los editores están planteándose la posibilidad de efectuar campañas publicitarias en aquellos medios que los jóvenes usan habitualmente, como son la televisión e Internet. Esta iniciativa, que se ha realizado ya en Francia ${ }^{11}$, permite a los diarios dar a conocer en televisión su orientación o

${ }^{11}$ Le Monde, 5-VI-2004. 
sus contenidos y no sólo anunciar sus promociones como habían hecho hasta ahora. De este modo, la prensa de pago puede comunicar lo que la distingue de los gratuitos, llegar a un público que se interesa poco por la prensa y optimizar las operaciones promocionales como viene haciendo en la actualidad.

Esto no deja de ser una medida paradójica, pues en la época dominada por los medios audiovisuales parece que la salvación de la prensa vendrá precisamente de sus competidores. Con todo, es una medida válida si consigue su objetivo de aumentar el consumo de prensa entre los jóvenes.

\section{REPENSAR EL PERIODISMO ESCRITO}

Llegados a este punto, veamos las estrategias de comunicación que algunos autores han sugerido para interesar a nuevas audiencias. La mayor parte de las propuestas plantean como tarea prioritaria la refundación ${ }^{12}$ del periódico o incluso la redefinición del periodismo como profesión ${ }^{13}$. En definitiva, se trata de someter el periódico a un proceso de reformulación, que consiste en elaborar un producto a la medida del lector. Un diario de servicios, menos interpretativo, personalizado y con más contenidos locales, e interactivo. Un diario hecho para que el lector lo seleccione, lo edite o lo reciba selectivamente ${ }^{14}$.

En segundo lugar, el proceso de refundación precisa de un plan de marketing en el que se analicen producto, precio, promoción y distribución en referencia a las publicaciones periódicas $^{15}$. En este sentido, algunos diarios han empezado a hacer encuestas telefónicas entre un target entre los 14 y 24 años, donde las preguntas claves se refieren a las portadas, el diseño, las secciones, las fotos, los suplementos. De este modo, se obtienen dos tipos de información: una sobre las costumbres de los consumidores -a partir de las cuales se podrían elaborar los contenidos-, y otra sobre la aceptación de los cambios que se van realizando en el periódico.

En relación al precio y a la distribución -donde la competencia de los gratuitos es cada día más fuerte-, la respuesta de la prensa diaria de información general pasa por acercar los productos a los lugares que frecuentan los menores de 30 años: cafeterías, centros culturales, gasolineras y centros comerciales, y ampliar el horario de distribución hasta bien entrada la noche. Al mismo tiempo, los periódicos diarios deberían promocionarse en ambientes juveniles e incluso infan-

\footnotetext{
${ }^{12}$ El profesor De Pablos habla de una necesaria refundación del periódico ante las amenazas desde el lado tecnológico, y de contenidos y conocimiento. "Estrategias informativas para acceder a un periodismo de calidad, en prensa y TV.”, en Ámbitos, nำ11 y 12, 2004, Sevilla.

${ }^{13}$ Es el caso de Martínez Albertos, por ejemplo en su obra El ocaso del periodismo, editorial CIMS 97, Barcelona, 1997.

${ }^{14}$ ROJO, P.A., Producción periodística y nuevas tecnologías. Estrategias para la prensa ante la convergencia mediática. Comunicación Social, Sevilla, 2003.

${ }^{15}$ Acerca de este aspecto Francisco Iglesias hace aportaciones interesantes en su libro Marketing periodístico.
} 
tiles, realizando para este fin ediciones especiales elaboradas por periodistas y pedagogos, pero también por redactores jóvenes a los que los editores han de dar un cierto grado de responsabilidad en el diseño de la agenda temática ${ }^{16}$.

Es preciso preparar a las generaciones jóvenes para que se sumen a la cultura del periódico en soporte papel como complemento de la inevitable cultura audiovisual y cultura digital. En este punto hay que tener claro que el futuro de la cultura escrita pasa por la complementariedad de medios, puesto que los jóvenes son usuarios habituales de Internet ${ }^{17}$. La mitad de los niños y adolescentes de nuestro país de entre 8 y 13 años se declaran internautas habituales, pero el porcentaje aumenta hasta el $60,5 \%$ en el tramo comprendido entre los 11 y los 13 años y se dispara hasta el $70 \%$ entre los 14 y los 18 años ${ }^{18}$.

No es fácil, desde luego, acometer la reformulación de la prensa de cara a los jóvenes consumidores. Son varios los aspectos que reclaman una revisión, pero, siendo todos ellos importantes, quizás sea en los contenidos donde haya que poner más imaginación.

\subsection{Gestión de contenidos para audiencias específicas}

Hace quince años los editores norteamericanos advirtieron que la estrategia pasa por nuevos lenguajes y nuevos contenidos, más próximos a las inquietudes y demandas de un segmento de la población al que no le interesa una información que califican de oficialista y cargada de contenido político. Quizás por esto la prensa deportiva y la prensa gratuita han logrado asentarse entre los jóvenes.

La fuerte penetración de la prensa deportiva revela la vigencia del soporte cuando el producto atiende las demandas específicas, incluso sobre sectores de población con un bajo poder adquisitivo. Los diarios deportivos encuentran su audiencia mayoritaria en la población juvenil: el 28,1 por ciento de los lectores de este producto son menores de 24 años, porcentaje que se dobla para la franja que llega a los 34 años: en este tramo de edad la prensa deportiva encuentra su público mayoritario ${ }^{19}$.

Por lo que hace a la prensa gratuita, la aparición del tabloide gratuito de The Washington Post, destinado a captar al público más joven, de entre 18 y 34 años, ha supuesto un auténtico boom y una interesante propuesta desde el sector de la prensa $^{20}$. El modelo de la prensa gratuita, con un formato de lectura rápida, textos cortos, imágenes de personas jóvenes en portada y contenidos atractivos y cercanos a la juventud, constituye una fórmula que ha triunfado en todo el mundo.

\footnotetext{
${ }^{16}$ LÓPEZ, M. op.cit, p.184

17 Ibidem, p.59

18 Estudio sobre la Audiencia Infantil/Juvenil de Medios en España 2004.

${ }^{19}$ Informe Anual de la Comunicación 1998-1999, p.19

20 "Los diarios alternativos se unen a los gratuitos y a los diarios para jóvenes como producto eficaz de captación de nuevas audiencias" en www.mediabriefing.com 2003.
} 
En realidad, la prensa gratuita ha generado un perfil de lectores bastante diferente al de los de pago, que incluye, además, una importante incorporación de mujeres y jóvenes ${ }^{21}$ urbanos, de ambos sexos, formados y activos. El atractivo de los gratuitos para la juventud reside en una oferta informativa variada, desenfadada y en pequeñas dosis, que requiere el mínimo esfuerzo intelectual y se consume en 20 minutos. $Y$ aunque es verdad que el modelo de la prensa gratuita está removiendo los cimientos de la industria tradicional y favoreciendo la reconversión de sus periódicos, no deja de ser un modelo cuestionado ${ }^{22}$ en cuanto a la calidad del producto.

En este aspecto, la renovación de contenidos que ha de acometer la prensa diaria no puede ser otra que una oferta de calidad $^{23}$, sobre todo teniendo en cuenta el periodo de formación en que se encuentra el público al que se dirige.

Varios autores han coincidido en señalar a los contenidos como causa primordial del bajo índice de lectura de periódicos entre los menores de 24 años. A los jóvenes no les interesan los periódicos porque no se sienten identificados con los temas que tratan ni con el modo como los tratan. Manuel López propone, por una parte, una renovación de contenidos basada en la búsqueda de temas emergentes que interesan a los jóvenes de hoy, por ejemplo, deporte, cine, empleo, consumo, secciones de entretenimiento, servicios multimedia. Y por otra, redactar las noticias con un lenguaje más cercano a los ambientes donde se encuentra la juventud.

Para Francisco Iglesias el cometido de las empresas periodísticas es descubrir nuevas demandas y nuevos intereses informativos cuando estos se abren camino en la vida social, lo que conlleva buscar fórmulas y procedimientos eficaces que respondan a esos deseos. Para lograr estos objetivos la prensa del futuro necesita ofrecer periódicos asequibles, fáciles de leer; con textos breves y concisos, espacios ordenados y bien distribuidos, y todo ello acompañado del material gráfico suficiente para ilustrar los textos ${ }^{24}$.

Allá por los años ochenta, Nicolás Negroponte apuntó la idea de los diarios personalizados con contenidos que respondan a los intereses específicos de los lectores. Para este autor el diario personalizado ofrece la ventaja de que cada lector recibe una edición única, hecha a su medida y distinta de todas las demás ${ }^{25}$.

\footnotetext{
${ }^{21}$ EGM 2003 indicaba que más del 50\% de los lectores de gratuitos son jóvenes menores de 34 años, de ellos un 26\% de edades entre 25 y 34 años, un 14\% entre 20 y 24 años y un 10,5\% tiene entre 14 y 19 años. Citado por BLANCO CASTILLA, E., "El desarrollo de la prensa gratuita en España: claves de un éxito", en Informe Anual de la Profesión Periodística 2004.

${ }^{22}$ Por profesionales del periodismo como Victor de la Serna: "Ni tanto, ni tal calvo" en Cuadernos de periodistas, nำ1, octubre, Asociación de la Prensa de Madrid.

${ }^{23}$ Sobre este punto, véase DE PABLOS, J.M., op.cit., p.341.

${ }^{24}$ IGLESIAS, F., Op. cit., p.72

${ }^{25}$ NEGROPONTE, N., "El futuro de la prensa y la prensa del futuro", Nuestro Tiempo, n $403-$ 404, enero-febrero 1988, p.35.
} 
Ahora bien, para la selección temática se necesitan hombres y mujeres jóvenes, más sensibles a las cuestiones que preocupan e interesan a la juventud. De este modo, el equipo de redacción se preocupará de que cuestiones objetivamente importantes no queden marginadas de la publicación, pero también de buscar aquellas otras que logran despertar un interés en el público ${ }^{26}$ de su generación.

Los periódicos están cada vez más centrados en el análisis político diario y los contenidos sociales de carácter local están desapareciendo o disminuyendo su peso en la agenda temática. Más o menos esa es la percepción de los jóvenes, que echan de menos más informaciones que "se puedan tocar", es decir, reclaman contenidos de proximidad $^{27}$.

Finalmente, conviene recordar que el periódico siempre ha sido un producto familiar. Es un hecho comprobable que el periódico lo compra uno pero lo leen varios. Aunque la presencia del diario en los hogares ha descendido, todavía es un producto de consumo familiar, en el que cada miembro busca la información que le interesa; sin que esto impida que hojee el resto de las páginas. Es un hecho comprobado que muchos jóvenes se han iniciado en el consumo de prensa a través de la sección de deportes del periódico que compraba el padre y luego han acabado leyendo las páginas de política. Manuel López propone un diario plurifascicular y pluritemático ${ }^{28}$,que satisfaga a diversas personas al mismo tiempo. ¿Se podría así fomentar el consumo de prensa desde edades muy tempranas? Hay que reconocer que no es una tarea fácil, pero hay que confiar, pues la creación de contenidos para audiencias específicas está dando buenos resultados en otros ámbitos, por ejemplo en el sector de la prensa gratuita.

\section{CONCLUSIONES}

- El consumo de prensa diaria entre los jóvenes va descendiendo año tras año desde 1997, como refleja la Asociación de Investigación en Medios de Comunicación (AIMC).

- Las causas son complejas: por un lado, la falta de convivencia con los diarios en los hogares; por otro, el mantenimiento de unos contenidos inadecuados $y$, finalmente, la influencia de las nuevas tecnologías.

- Las empresas periodísticas y varios periódicos generalistas han puesto en práctica diversas iniciativas que no han logrado aumentar el consumo de prensa diaria, ya que los suplementos y páginas juveniles se perciben como un subproducto, que no necesariamente crea afición por el periódico de referencia.

${ }^{26}$ IGLESIAS, F., op.cit, p.73

${ }^{27}$ SABÉS, F., "Jóvenes y medios: ¿encuentro o desencuentro?" en Cuadernos de periodistas, no5, diciembre, 2005, Asociación de la Prensa de Madrid.

${ }^{28}$ LÓPEZ, M., op.cit, p.102. 
- Según varios expertos, el periodismo está en crisis, no sólo entre los jóvenes. Para ellos lo que necesita la prensa diaria es una reformulación del periódico creando un producto a la medida del lector.

- Este proceso comenzaría con un plan de marketing de la prensa, que atienda al precio, la promoción y la distribución del producto, siempre teniendo en cuenta los hábitos de los jóvenes.

- Y proseguiría con una renovación de contenidos y de lenguaje más próximo a este segmento de la población. La fidelidad de la audiencia juvenil de los diarios deportivos y el éxito de la prensa gratuita entre los jóvenes revelan la vigencia de un soporte cuando atiende a las demandas de su público.

- El diario del siglo XXI se perfila como un periódico personalizado, pluritemático y plurifascicular, que pueda ser utilizado por todos los miembros de la familia.

\section{BIBLIOGRAFÍA}

Audiencia Infantil/Juvenil de Medios en España 2004, Madrid, AIMC.

BLANCO CASTILLA, E: "El desarrollo de la prensa gratuita en España: claves de un éxito" en Informe Anual de la Profesión Periodística 2004, Asociación de la Prensa de Madrid.

DE PABLOS, J.M: "Estrategias informativas para acceder a un periodismo de calidad, en prensa y TV.", Ámbitos, №11 y 12, 2004, Sevilla.

DÍAZ NOSTY, B: Informe Anual de la Comunicación 1999-2000. Estado y tendencias de los medios en España, Ediciones Zeta.

DÍAZ NOSTY, B: Informe Anual de la Comunicación 2000-2001. Estado y tendencias de los medios en España, Ediciones Zeta.

DíAZ NOCI, J., MESO, K: Periodismo en Internet. Modelos de la prensa digital, Universidad del País Vasco, Bilbao, 1999.

EDO, C: Del papel a la pantalla. La prensa en Internet, Comunicación Social, Sevilla, 2002.

IGLESIAS, F: Marketing periodístico, Ariel Comunicación, Barcelona, 2001.

Informe Anual de la Profesión Periodística 2004, Asociación de la Prensa de Madrid. Libro Blanco de la Prensa Diaria 2005, Asociación de Editores de Diarios de España. LÓPEZ, M: Nuevas competencias para la prensa del siglo XXI, Paidós, Barcelona, 2004. MARTíNEZ ALBERTOS, J. L: El ocaso del periodismo, Editorial CIMS 97, Barcelona, 1997.

NEGROPONTE, N: "El futuro de la prensa y la prensa del futuro", Nuestro Tiempo, no 403404, enero-febrero 1988, Pamplona. 
ROJO, P. A: Producción periodística y nuevas tecnologías. Estrategias para la prensa ante la convergencia mediática, Comunicación Social, Sevilla, 2003.

www.wan-press.org

www.mediabriefing.com

(Recibido el 20-3-06, aceptado el 3-5-06) 